\title{
Towards a reliable bridge collision warning system for inland vessel navigation based on RTK height determination
}

\author{
Heßelbarth, A.; Ziebold, R. ${ }^{1}$; Sandler, M. ${ }^{2}$, Alberding, J.; Uhlemann, M. ${ }^{3}$; Hoppe, M.; Bröschel, M. ${ }^{4}$; \\ ${ }^{1}$ German Aerospace Center, Institute for Communication and Navigation, 17235 Neustrelitz, Germany \\ anja.hesselbarth@dlr.de \\ ${ }^{2}$ in - innovative navigation GmbH, 70806 Kornwestheim, Germany \\ ${ }^{3}$ Alberding GmbH, 15745 Wildau, Germany
}

${ }^{4}$ FVT, German Federal Waterways and Shipping Administration, Koblenz, Germany

\begin{abstract}
Inland shipping is an important pillar of the European transport system; however the challenges of inland navigation due to dense traffic, increasing ship dimension, reduced maneuver space and visibility are multifaceted and increasing. About 20 to 30 collisions per year, partially caused by carelessness, demonstrate the necessity of driving assistance systems for inland waterway applications.

The project LAESSI (Guiding and assistance systems to improve safety of navigation on inland waterways) is developing efficient navigation assistance functions for inland waterway transport which aim the reduction of risk of collisions by supporting the skipper in its task. Therefore, nautical information like position, height, velocity and heading has to be determined. One focus in this contribution is a bridge collision warning system, which provides a timely alert to the skipper, whenever the vessel, particularly the wheelhouse or radar mast, will not safely pass the bridge.

A feasibility study has identified Global Navigation Satellite System (GNSS) technologies as basis for the reliable height determination for such a bridge collision warning system. This approach requires information about the vertical clearance of the bridge superstructure as well as precise height information in the same height reference system at least $300 \mathrm{~m}$ before the vessel will pass the bridge. The high accuracy level of less than $10 \mathrm{~cm}$ in the vertical position necessitates the Real Time Kinematic (RTK) as suitable GNSS processing method.

RTK is a phase-based differential GNSS technique and uses additional observations from permanent reference stations to mitigate or eliminate effects like tropospheric and ionospheric delays or satellite clocks and orbit errors. As bridge collision warning system is a safely critical application, this means that beside the provision of accurate height information also an integrity monitoring for the complete RTK procedure has to be introduced. The shore-based architecture is designed in such a way that correction data, based on a permanent GNSS reference station network, will be checked for their integrity before they are broadcasted (Pre-Broadcast Monitoring) to the processing computer on the vessel.

Furthermore, several station dependent effects like signal interferences, signal losses or multipath effects, caused due to obstacles near the inland waterways, impact the GNSS signals, which hamper or falsify the ambiguity fixing and finally the position results. To prevent the provision of faulty results a further integrity monitoring for the shipborne architecture has to be applied. This shipborne integrity monitoring utilizes on the one hand a number of internal statistical parameters from the RTK algorithm like the conventional used fixed ratio value and on the other hand additional information from other sensors or observations.
\end{abstract}


The contribution will present the derived requirements for accuracy, integrity and time to alert for different inland waterway assistance functions. Furthermore an overview about the system architecture and a communication concept based on VHF Data Exchange System (VDES) communication channels will be given. The main focus, however, is an RTK-algorithm which not only estimates position, velocity and heading, but also provides integrity information. This concept considers in a first realization internal statistical parameters from the RTK adjustment, other observations (e.g. Doppler shift) and physical information (e.g. baseline length of two GNSS antennas).

A first measurement campaign was done in July 2016 in Koblenz/Germany on the river Moselle. This test area is characterized by three bridges in a relatively short distance of only $300 \mathrm{~m}$ which make the provision of reliable and precise RTK position data rather challenging. The preliminary analyses based on three hours GPS and GLONASS observations showed a high rate (> 85\%) of evaluated position and height results, which fulfilled the required cm-level accuracies. The first implementation of the internal integrity check could detect and eliminate faulty results. The results have shown that beside a good height performance also fast provision of the heights after the vessel sailed through the bridges is recognizable. This position data between the bridges allows a provision of information to the skipper to check or adjust the wheelhouse or radar mast height before the next bridge will be passed.

In the next implementation the rate of turn and acceleration data from an Inertial Measurement Unit (IMU) will be tightly coupled with the raw GNSS observations. Thereby additional information will be available to evaluate the position, height, velocity and heading results and increase the integrity validation. Furthermore it can be expected that the ambiguities after a complete signal interruption can be fixed faster.

Summarized this contribution will present high accurate results, based on a RTK-algorithm, together with integrity validation which can be used for example as part of a bridge collision warning system on inland waterways. A large number of collisions per year demonstrate the necessity of such kind of support to the skipper in its tasks of guiding a vessel.

\section{What is new or innovative?}

The height determination for the bridge collision warning system on inland waterways with rather challenging conditions applies, instead of the usual for navigation used code-based technique, the more precise phase-based RTK GNSS processing method to provide results with a high accuracy level. Furthermore a comprehensive integrity concept on the shore-based as well as on the ship-borne side will evaluate the obtained results to prevent the provision of faulty results.

\section{Session:}

Marine Application 